\title{
HOUSING IN MOSAMBIK - PLURALITÄT \\ IM MOSAMBIKANISCHEN KINO
}

\author{
HOUSING EM MOÇAMBIQUE - \\ PLURALIDADE NO CINEMA MOÇAMBICANO
}

\author{
HOUSING IN MOZAMBIQUE - \\ PLURALITY IN MOZAMBICAN CINEMA
}

Kathrin Sartingen ${ }^{1}$

Sophie Baltas ${ }^{2}$

\begin{abstract}
,Wohnen' stellt immer noch ein Thema dar, das in Bezug auf den Film weitgehend unbehandelt scheint. Obwohl Düllo (2008, p. 358) in seinem Artikel Wohnen im Film feststellt, dass die jeweiligen Wohnsituationen von Filmfiguren ausschlaggebend für deren Verortung im Leben und Handeln bzw. deren Verankerung in der diegetischen Handlungsebene und -aktion sind, wird der spezifischen Wohnraumgestaltung bislang sehr wenig Bedeutung beigemessen. Dabei sind es gerade Aspekte wie die räumliche Verortung der ProtagonistInnen im Wohnraum sowie das individuelle Ausgestalten der Wohnräume - das spacing (DÜLLO, 2008, p. 369f) -, die essentielle und für das Filmgeschehen signifikante Aussagen zur kulturellen, sozialen, psychischen und identitären Einbettung der Figuren in konkrete Kontexte und Räume liefern. Insofern repräsentieren filmisch abgebildete Wohnungen, Häuser, Orte und Nicht-Orte (AUGÉ, 1994) immer auch die diversen kulturellen und geopolitischen Kontexte, in denen sich die Filmfiguren aufhalten. Sie lassen Rückschlüsse auf individuelle bzw. kollektive Wohnkulturen zu, und damit auf den Status, den Wohnraum in den verschiedenen Kulturen innehat, sowie die Möglichkeiten, Wohnraum zu haben, zu erhalten, auszugestalten, oder auch nicht zu haben. Am Beispiel von zwei mosambikanischen Dokumentarfilmen, Hóspedes da Noite von Licínio Azevedo (2007) und Grande
\end{abstract}


Hotel von Lotte Stoops (2010) soll die diversifizierte Wohnsituation im alten Kolonial-Hotel in Beira, wie sie sich in den beiden Filmen spiegelt, analysiert werden. Im Kontinuum zwischen Spielfilm und Dokumentation angesiedelt, werden neue Perspektiven auf die Verhandlung von, Wohnen' in kolonialer und postkolonialer Zeit, und damit auf die (Re-)Vision von Vergangenheit und Gegenwart in Mosambik präsentiert.

SCHLÜSSELWÖRTER: Wohnraum. Kulturelles Gedächtnis. Doku-Fiktion. Mosambik.

\section{RESUMO}

O "habitar" ainda representa um tópico que parece em grande parte não abordado na análise de filmes. Embora Düllo (2008, p. 358) declare em seu artigo "Wohnen im Film" ("Viver no Cinema") que as respectivas situações de moradia dos personagens fílmicos são cruciais para situá-las em suas vidas e em suas ações, isto é, determinar sua ancoragem no nível diegético da ação, muito pouca importância tem sido dada até agora à específica configuração do espaço habitado pelos personagens. No entanto, é precisamente o aspecto da localização espacial de protagonistas no seu espaço habitacional que fornece informações significativas a propósito da incorporação cultural, social, psicológica e identitária dos personagens em contextos e espaços concretos. Neste sentido, os apartamentos, casas, lugares e não-lugares (AUGÉ, 1994) cinematograficamente representados refletem sempre os diversos contextos geopolíticos nos quais os personagens do filme se encontram. Eles permitem tirar conclusões sobre culturas habitacionais individuais ou coletivas e, portanto, sobre o estatuto que o espaço habitado tem nas diversas culturas, bem como as possibilidades de ter, manter, ou mesmo não ter moradia, isto é, um espaço para habitar. Tomando como exemplos dois documentários moçambicanos, Hóspedes da Noite de Licínio Azevedo (2007) e Grande Hotel de Lotte Stoops (2010), serão analisadas as diferentes dimensões do habitar no antigo hotel colonial em Beira. Situadas na interface entre longa-metragem e documentário, serão apresentadas novas perspectivas sobre a negociação das formas do "habitar" nos tempos coloniais e pós-coloniais, bem como sobre as formas de (re)visão do passado e do presente em Moçambique.

PALAVRAS-CHAVE: Espaço habitacional. Memória cultural. Docuficção. Moçambique.

\section{ABSTRACT}

'Housing' still represents a topic that seems to be largely unaddressed in relation to film. Although Düllo (2008, p. 358) states in his article Wohnen im Film that the respective living situations of film characters are decisive for their localisation in life and action i.e. their anchoring in the diegetic levels, the specific design of living space has so far been regarded as of little importance. Yet, it is precisely aspects such as the localisation of the protago- 
nists in the living space as well as the individual design of these spaces - the spacing (DÜLLO, 2008, p. 369f) - that provide essential information about the way in which they find themselves embedded culturally, socially, psychologically and by way of identity within the film. In this way, film viewers gain significant insights into the characters' lives and development. Besides the situational insights into the life of the various individuals represented, films make concrete and visible statements about the cultural contexts of the characters, regions, spaces and countries shown by means of the staging of living situations. Films depict different housing cultures. Thus, depicted flats, houses, places and non-places (AUGÉ, 1994), i.e. all the different 'living' situations medialised in films, represent the diverse cultural and geopolitical contexts in which the film characters reside. The staged living spaces allow conclusions to be drawn about individual or collective living cultures, i.e. about the status that a living space carries in various cultures and the possibilities of having, maintaining, designing or not having living space.

KEYWORDS: Housing. Cultural Memory. Docufiction. Mozambique.

Der mosambikanische Film ist reich an Beispielen für das hier zu erörternde Thema des housing. Seine Vielfalt in dem Bereich speist sich aus einer bemerkenswerten filmästhetischen Besonderheit: Er , behandelt' dieses Thema nicht nur im Spielfilm; vielmehr liegen besonders eindrucksvolle Beispiele in der Gattung des Dokumentarfilms vor. Und dabei geht es nicht um das Thema,Wohnen' als intrinsischer Untersuchungsgegenstand des Films (Stichwort „explizites Wohnen“, DÜLLO, 2008, p. 361), sondern um Dokumentationen, die sich möglicherweise einem ganz anderen thematischen Zusammenhang widmen, aus denen sich aber zahlreiche, sehr auffällige Rückschlüsse auf die Wohn-Kultur der jeweils abgebildeten Zeit und des kulturellen Umfeldes ableiten lassen. Dieser sogenannte implizite Auftritt des Wohnens ist laut Düllo (2008, p. 361) in jedem Film präsent und auch der ertragreichere für die Analyse, da er sich näher an der Lebensrealität der Zuschauer:innen bewegt. Diese Form der Wohnrauminszenierung wirkt authentischer und unterstreicht eben dadurch den soziokulturellen Kontext, in dem die Figuren verortet sind. In den Fällen, in denen das Wohnen ,auffälliges und signifikantes (Sub-)Thema ist“ (DÜLLO, 2008, p. 361), kann die Analyse nicht nur auf bestimmte Wohn-Kulturen schließen, sondern über das Sujet Wohnen und die filmisch dargestellten Räumlichkeiten tieferliegende gesellschaftliche Fragestellungen thematisieren.

Das bedeutet, dass Wohnraum und Einrichtung eine narrative Funktion übernehmen, die innerhalb der Filmtexte identifiziert werden kann. Laut Düllo (2008, p. 366f) stellt die Wohnpraxis einen soziokulturellen Prozess dar, der im Film immer doppelwertig gelesen werden muss - unter Berücksichtigung sowohl raum- als auch filmtheoretischer Kategorien. Die Analyse des Wohnens im Film soll idealerweise die Untersuchung filmischer 
Darstellungsstrategien mit der des räumlichen sowie gesellschaftlichen Umfelds kombinieren, um der Funktion und Bedeutung des Wohnens im Film auf den Grund zu gehen. Die Aneignung einer solchen Perspektive scheint insbesondere naheliegend in der Auseinandersetzung mit Filmen, die sich im Spannungsfeld zwischen Spielfilm und Dokumentation bewegen, da in diesen Fällen - wie im Folgenden gezeigt werden wird - der Realitätsbezug, den die Wohnrauminszenierung etabliert, zusätzlich instrumentalisiert wird.

In Anlehnung an Düllo soll in Folge am Beispiel von zwei Dokumentarfilmen, Hóspedes da Noite von Licínio Azevedo (2007) und Grande Hotel von Lotte Stoops (2010), die diversifizierte Wohnsituation in Beira, wie sie sich in den beiden Filmen spiegelt, analysiert werden. Beide Filme liegen gattungstypologisch im Kontinuum zwischen Spielfilm und Dokumentation, wodurch sie dem Publikum nicht nur über die Inszenierung des Sujets, sondern ebenso auf ästhetischer Ebene neue Perspektiven auf die Verhandlung von,Wohnen' in kolonialer und postkolonialer Zeit, und damit auf die (Re-)Vision von Vergangenheit und Gegenwart bieten. Dabei bedienen sie sich verschiedener narrativer Mittel, um unter visuellem und narrativem Einbezug der Räumlichkeit(en) des Hotels die Geschichte des Landes aufleben zu lassen, indem die im Hotel verankerten Erinnerungen an koloniale Dekadenz mit dem Überlebenskampf der heutigen Bewohner kontrastiert werden. Durch das Umherschweifen in den Schichten dieser Erinnerungen gelingt es Azevedo wie auch Stoops, die Nachwehen von Kolonialzeit, Unabhängigkeitskrieg und Bürgerkrieg, die bis heute deutlich zu spüren sind, filmisch zu inszenieren und die Geschichtlichkeit des Ortes in einen logischen Zusammenhang mit seiner Aktualität zu bringen. Es kommt dabei in beiden Filmen zu einer Art Individualisierung der kollektiven Geschichte: indem auf visueller wie auch narrativer Ebene ein Mosaik an persönlichen Geschichten inszeniert wird, gelingt es den Regisseuren, das kollektive, nationale Gedächtnis und damit die Vielschichtigkeit der mosambikanischen Vergangenheit und Gegenwart filmisch zu vermitteln.

\section{DIE GESCHICHTE DES GRANDE HOTEL}

Das Grande Hotel in Beira wurde im Jahr 1955 eröffnet; es entsprang der Megalomanie des kolonialen Regimes sowie deren Baubeauftragten und wurde finanziert durch die Companhia de Moçambique (GRANDE HOTEL, 2007). Dabei sollte das Hotel die Idee eines tropischen Eden an der Küste des Indischen Ozeans propagieren; ein Bild, das von vielen Touristen mit der geplant urbanisierten, kosmopolitischen und multikulturellen Hafenstadt Beira in Verbindung gebracht wurde (SPINUZZA, 2018, p. 162f). Doch durch die maßlosen Forderungen des Projektleiters verlief sich das Bauvorhaben schon bald in seiner eigenen Dekadenz. Das Resultat war eine Hotelanlage mit übertriebenen Dimensionen, deren laufende Kosten von den im Verhältnis wenigen Zimmer nicht gedeckt werden konnte. Schon 1962 musste das Hotel für (fast) immer seine Türen schließen.Nachdem es während des Bürgerkriegs 
als Militärstützpunkt und -gefängnis genutzt wurde, kamen im Jahr 1981 die ersten Flüchtlinge im ehemaligen Hotel an, um dort Unterschlupf und vor allem auch eine Art von Sicherheit und Beständigkeit zu finden. Ohne Perspektive auf einen anderen, dauerhaften Wohnraum transformierten die Menschen das improvisierte Flüchtlingslager in eine konstante Wohnstätte (PUTSCH, 2010). Zur Zeit der Dreharbeiten und auch heute noch war und ist das Hotel eine Ruine, vielfach ohne Strom und ohne fließend Wasser. Von den bis zu 3.000 Personen ${ }^{3}$, die sich seither dort einquartiert haben, leben einige schon seit über 30 Jahren in den Zimmern und Gängen des Hotels, wodurch sich eine mittlerweile generationenübergreifende Mikrogesellschaft gebildet hat.

Der Beheimatungsprozess im Grande Hotel - einem Übergangsort par excellence (AUGÉ, 1994, p. 92f) - scheint für Außenstehende eine unmögliche Unternehmung zu sein. Die Wohnsituation der im Hotel angesiedelten Grenzgesellschaft ist, trotz der verschiedensten Anstrengungen, sich permanent einzurichten, vergleichbar mit jener in Flüchtlingslagern bzw. - unterkünften. Diese werden nach Augé (1994, p. 64-67) als Nicht-Orte kategorisiert, da sie für den Übergang, nicht für die Verortung der darin „aufgefangenen“ Personen konzipiert sind und demnach nicht den passenden Rahmen für die Einschreibung von Identität, Relation und Geschichte bieten. Den Bewohner:innen wird die Beheimatung erschwert, da sie sich weder räumlich noch emotional dauerhaft verorten können, was dazu führt, dass sie sich mental in einem Zustand des ständigen Transit befinden.

Dieser Schwebezustand ist Ergebnis unterschiedlicher Faktoren, darunter fehlende Ressourcen und Infrastrukturen sowie nicht zuletzt ein beträchtlicher Mangel an Sicherheit, der durch die Auflösung von Innen- und Außenraum, privater und öffentlicher Sphäre verstärkt wird. Für Bollnow $(1963,1970)$ ist ein Wesenszug des vom Menschen gelebten und erlebten Raums die Gliederung in einen bewohnten Innenraum, der ihm Sicherheit und Geborgenheit bietet, und einen bedrohlichen, unheimlichen Außenraum, den er beim Verlassen seiner Wohnung betritt. Ein Grundbedürfnis des Menschen ist daher die Verwurzelung durch das Wohnen, um sich einen Bereich zu schaffen, mithilfe dessen er sich vor den Bedrohungen der Außenwelt schützen kann. Im Spannungsfeld zwischen Innen und Außen, Privat und Öffentlich zeigt sich demnach die Verletzbarkeit des wohnenden Menschen, aber auch sein Trieb zur Beheimatung (GESCHKE, 2013).

Die außergewöhnliche und facettenreiche Wohnsituation im Grande Hotel bietet eine Vielzahl an Inszenierungsmöglichkeiten, die es - wie bereits erwähnt - zulassen, über das Sujet Wohnen Rückschlüsse auf Wohnkulturen zu ziehen, und u.a. über die filmische Darstellung tieferliegende gesellschaftliche Prozesse zu diskutieren. In beiden Produktionen ist eine eindeutig politisch sowie sozial motivierte Intention zu erkennen. Während Stoops ihren Blick auf die Einflüsse der Politik auf das Privatleben wirft, fokussiert Azevedo das kollektive Gedächtnis der Nation und richtet den Blick mehr auf die transformativen (Über-) Lebensstrategien der Bewohner:innen (SPINUZZA, 2016, p. 281). Sowohl Stoops als auch Azevedo bewegen sich dabei 
inhaltlich wie ästhetisch ständig zwischen (vermeintlichen) Antagonismen: zwischen Dokumentation und Spielfilm, Vergangenheit und Gegenwart, Memoria und Vorausschau, Privatheit und Öffentlichkeit, Innen und Außen.

Da die Darstellung des Grande Hotel und seiner Räumlichkeit an vielen Stellen expliziter wirkt als das Wohnen in anderen Filmen, vermittelt die mediale Inszenierung der Räume sowie deren Einrichtung dem $\mathrm{Zu}$ schauer ein „Mehr“ an Information über die Bewohner, ihre Identität, ihre individuelle sowie die nationale Geschichte und das kollektive Gedächtnis Mosambiks. Folgt man Düllo (2008), gelingt diese Vermittlung u.a. auf Grund zweier Qualitäten des Wohnsujets im Film: zum einen durch die den Zuschauer:innen gebotene Vergleichsoberfläche (DÜLLO, 2008, p. 371), deren Effekt durch den dokumentarischen Charakter der beiden Filme verstärkt wird, zum anderen durch die Spiegelung des mentalen Programms der Figuren (DÜLLO, 2008, p. 362).

\section{FIKTION UND DOKUMENTATION: WOHNEN ALS VERGLEICHSANGEBOT}

Die effektive Vermittlung gesellschaftlicher Diskurse über das Sujet des Wohnens speist sich unter anderem aus dem Identifikationsangebot, das den Zuschauer:innen zur Verfügung gestellt wird. Denn nicht nur im Film, sondern auch in der extradiegetischen Wirklichkeit ist das Wohnen omnipräsent - selbst wenn es sich um den Verlust oder das Fehlen von Wohnraum handelt. Insofern entwickelt der Wohnraum, das Innen, laut Düllo (2008, p. 361), durch das Vorhandensein dieses Vergleichsangebots mehr „identifikatorische Kraft“, als es das Außen vermag. Dabei spielt das sogenannte spacing eine Rolle, das den Zuschauer:innen den geeigneten Rahmen bietet, die Figuren und ihr Handeln einzugrenzen, um sie damit letztlich einordnen, das heißt, typisieren zu können. Das bedeutet, dass die Betrachter:innen über die individuelle Einrichtungs- und Geschmackspraxis der Figuren im Film den soziokulturellen Kontext ablesen können; gleichzeitig können sie diese Teilwelten mit der eigenen Realität vergleichen, sich in den Bildern wiederfinden oder sich von ihnen abgrenzen (DÜLLO, 2008, p. 369-371). Die Berücksichtigung des Kenntnis- und Erwartungshorizonts des Publikums in Bezug auf die soziokulturelle Praxis „Wohnen“ erzeugt einen Realitätseffekt (cf. AUMONT 1990) ${ }^{4}$, der auf dem Kriterium der Wahrscheinlichkeit beruht (DÜLLO, 2008, p. 371).

Einen großen Beitrag zu dieser identifikatorischen Wirkung, die beide Filme auf der Rezeptionsseite entfalten, leistet neben der Wahl der impliziten Inszenierung von Wohnraum auch ihr dokumentarischer Charakter. Indem die Abbildung der Wohnsituation als Ausgangspunkt genommen wird, gelingt es mithilfe genrespezifischer, eben dokumentarischer Darstellungsmethoden nicht nur, den Realitätseffekt zu verstärken, sondern auch die Wurzeln der prekären Situation, in denen sich die Bewohner:innen befinden, darzulegen. 
In der Filmwissenschaft wird längst keine klare Trennlinie mehr gezogen zwischen Dokumentation, als möglichst „exakter“, „objektiver“ Realitätsabbildung, und dem fiktionalen Spielfilm. Denn die filmischen objets trouvés der Anfänge des Kinos (wie etwa La sortie des usines Lumière, 1895) haben keineswegs die Bedeutung, die dem Dokumentarfilm heute zukommt (HATTENDORF, 1994, p. 44f). Die Bestimmung des Dokumentarischen berücksichtigt daher in erster Linie nicht das, „[...] was real ist, sondern was vom Rezipienten als , authentisch anerkannt wird." (HATTENDORF, 1994, p. 46). Der Grad der Authentifizierung - und damit der Einsatz bestimmter Erzähl- und Montagestrategien - hängt vom jeweiligen Repräsentationsanspruch (was soll dargestellt werden?) ab, da die Authentizität der Bilder Einfluss darauf hat, wie das Publikum das Verhältnis zwischen Inhalt und Wirklichkeit interpretiert (MUNDEHENKE, 2017, p. 18). Der Aspekt des Authentischen als Gradmesser für Glaubwürdigkeit (HATTENDORF, 1994, p. 62) ist daher ein ausschlaggebender Faktor, da durch den Einsatz von bestimmten Authentizitätsmerkmalen (z.B. beobachtende Kamera bzw. living camera, Einsatz von originalem Bild- und/oder Tonmaterial, Interviews, Auftritt von Zeugen, Verzicht auf Kommentare u.v.m.) die Ästhetik des Dokumentarischen hervorgehoben und dadurch die Erwartungshaltung sowie letztlich die Wahrnehmung des Zuschauers gelenkt werden können (MUNDHENKE 2017, p. 78). Die dadurch entstehenden Doku-Fiktionen (BLUM 2013) oder Doku-Hybride fußen auf diesem archaischen Antagonismus von Fiktion und Dokumentation bzw. perpetuieren ihn sogar, um mit der Erwartungshaltung des Publikums zu spielen und dadurch bestimmte „Lektüremodi“ zu aktivieren (HEIßNAUER, 2010, p. 17f; 27). Denn das vom Publikum interpretierte Verhältnis der Diegese zur Wirklichkeit entscheidet schlussendlich auch darüber, ob der vom Produzenten intendierte Subtext erfolgreich vermittelt wird.

In beiden Filmen kommen einige dieser Authentizitätsmerkmale zum Einsatz. Inhaltlich zeichnen sie sich insbesondere durch die Integration von originalem Bild- und Tonmaterial, Erzählungen und Interviews aus, die vom Film gezeigt bzw. von der Kamera begleitet werden, wobei auf Kommentare seitens der Produktion verzichtet wird. Die Erklärungen werden auf das Einblenden von Beschreibungen (wie etwa Name und Alter der Interviewten: z.B. GRANDE, 2010, 00:07:30 bzw. HÓSPEDES, 2007, 00:05:34) reduziert, welche den Verzicht auf einen Kommentar aus dem Off zusätzlich betonen. Dadurch wird die Kamera zu einem reinen Beobachter, streift auf eigene Faust durch die Gänge oder folgt den jeweiligen „Fremdenführern“: in Hóspedes da Noite (2007) sind es die beiden ehemaligen Hotelangestellten, Sr. Caíto und Sr. Pires, in Grande Hotel (2010) führt Moises die Kamera und damit die Zuschauer:innen durch das baufällige Hotel. Die dokumentarische Kamera eignet sich aber nicht nur für die schlichte Beobachtung, sondern ermöglicht es dem Publikum zudem, in fremde Lebenswelten einzudringen. Durch den voyeuristischen Blick über Schultern, durch Vorhänge und Türspalten (z.B. HÓSPEDES, 2007, 00:18:15; 00:19:21), werden den Zuschauer:innen 
Räume eröffnet, die der Öffentlichkeit normalerweise verschlossen bleiben. Dieser teils sogar heimlich beobachtende Duktus rückt den filmischen Produktionsprozess in den Hintergrund und verleiht den gezeigten Bildern zusätzlich Authentizität.

\section{DIE SPIEGELUNG DER MENTALEN VERFASSUNG VON FIGUREN}

Das von den Filmen zur Verfügung gestellte Vergleichsangebot in Verbindung mit der Darstellungsweise spielt eine zentrale Rolle für die Wirkung, die die Filme entfalten bzw. das Interpretationsangebot, das sie für ihr Publikum bereithalten. Sowohl Wohnraum als auch dokumentarischer Charakter werfen einen Anker in unsere Realität(-svorstellung) und bringenden Zuschauer dazu, die Figuren als Teil der eigenen Wirklichkeit und damit in einem festen soziokulturellen und geopolitischen Kontext zu sehen. Letztlich geht es, laut Düllo $(2008,360)$, „auch um den Vertrag zwischen Film und Rezipient, sich temporär während des Filmsehens und per Gedächtnis in den Wohn-Welten zu verorten, [...] sie vergleichend an die eigene Lebenswelt anzukoppeln $[\ldots]$...

Mithilfe dieses Vertrags gelingt es den Zuschauer:innen, das mentale Programm der Figuren abzulesen und zu interpretieren. Erstaunlicherweise ist es gerade deren psychische Verfassung, die wohn-räumlich ausgedrückt wird (DÜLLO 2008, p. 365 sowie HICKETHIER 2012:72). Die momentane Stimmung wie auch das zugrundeliegende mentale Allgemeinbefinden einer Figur spiegelt sich demnach in der Gestaltung des Wohnraums wider. Oft wird mithilfe der Wohnrauminszenierung eine Schwellensituation, d.h. eine Entscheidungssituation gezeigt, denn dem Wohnraum, laut Düllo (2008, p. 365f) wohnt eine "Spannungs- und Aktionslatenz“ inne, die ihn als Be- oder Entschleuniger der Handlung fungieren lässt.

Authentizität der Bilder und die Vergleichsoberfläche „Wohnraum“ verstärken die Nachvollziehbarkeit der räumlichen wie mentalen Schwellensituation, in der sich die Bewohner:innen des Nicht-Orts Grande Hotel befinden. Zum einen wird über die Verhandlung des Sujet Wohnen die Spannungslatenz des bewohnten Innenraums und damit der wohnliche Schwebezustand betont. Das Hotel, das einem Flüchtlingslager gleicht, wird zum Warteraum für all diejenigen, die vor Krieg und Hunger in die Stadt flüchten und den unweigerlichen Einsturz sowie ihre ungewisse Zukunft hinauszögern. Zudem operieren Hóspedes da Noite (2007) und Grande Hotel (2010), so wie viele andere Filme, „an der Bedrohlichkeit und Verletzbarkeit dieser Innen-/Außenraumgrenze" (Düllo 2008:368), indem sie die Verwischung der räumlichen Grenzen inszenieren und damit die prekäre Situation der Protagonisten ganz gezielt spiegeln.

\section{DER BEREICH DES LIMINOIDEN}

In Verbindung mit der Spiegelung der Verfassung steht auch der von Düllo (2008, p. 367) sogenannte Bereich des Liminoiden (bzw. der liminoiden Grenzen), der in den Filmen eine wesentliche Rolle spielt. Das Liminoide beschreibt den schwellenhaften Zwischenbereich zwischen Innen- und Au- 
ßenraum und umschreibt Bereiche, die von anderen Raumtheoretiker:innen (vgl. u.a. Rolshoven, Borsò, Kanne, Benjamin) auch als Übergänge, TransitRäume, Zwischenräume, Durchgänge, Schwellen und Passagen bezeichnet werden. Dabei handelt es sich um Fenster, Türen, Öffnungen, aber auch Flure, Gänge und Treppen. Das Liminoide kann, in Form von Spiegeln oder „digitalen Fenstern zur Welt“, wie dem Fernseher oder Radio (Düllo 2008:367f), zudem den Innenraum selbst durchziehen.

Durch das Fenster kann man - von der Sicherheit des Innen aus - das Außen beobachten; es stellt eine Beziehung zwischen Innen- und Außenwelt her. Was bedeutet es aber, wenn das Fenster fehlt, wenn es nur mehr ein Loch, eine Leerstelle gibt?

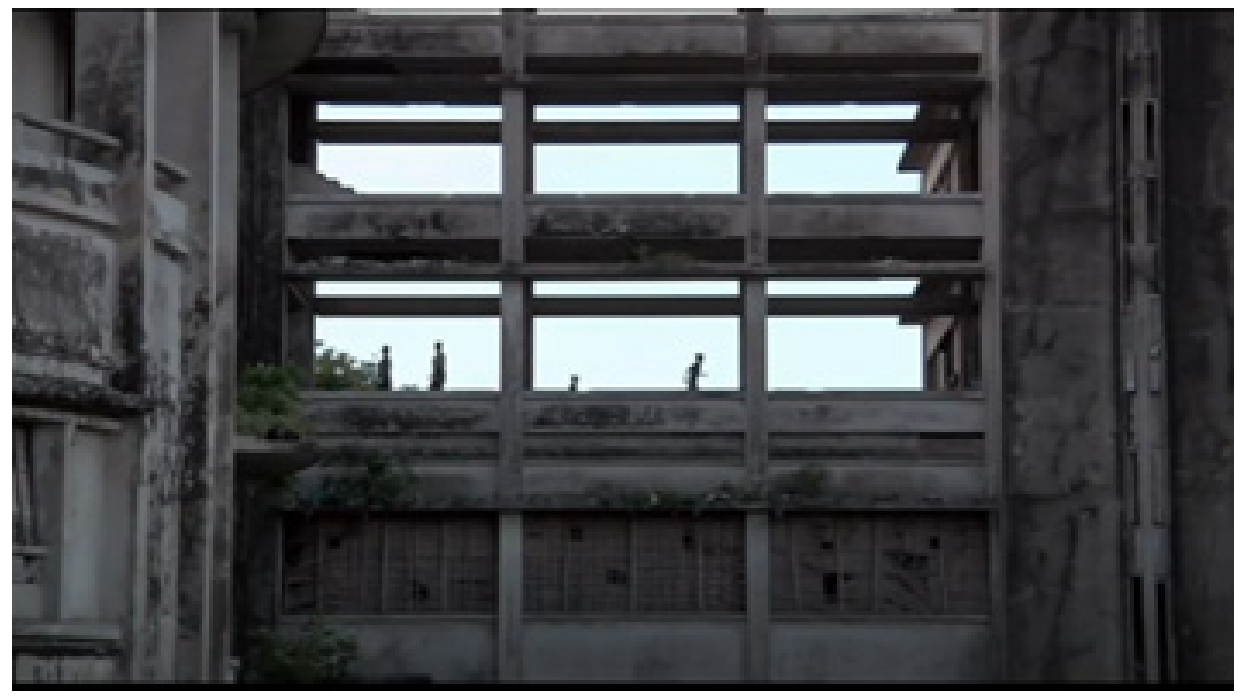

Abb. 1: Fehlende Fenster I.

Quelle: GRANDE, 2010, 00:09:02

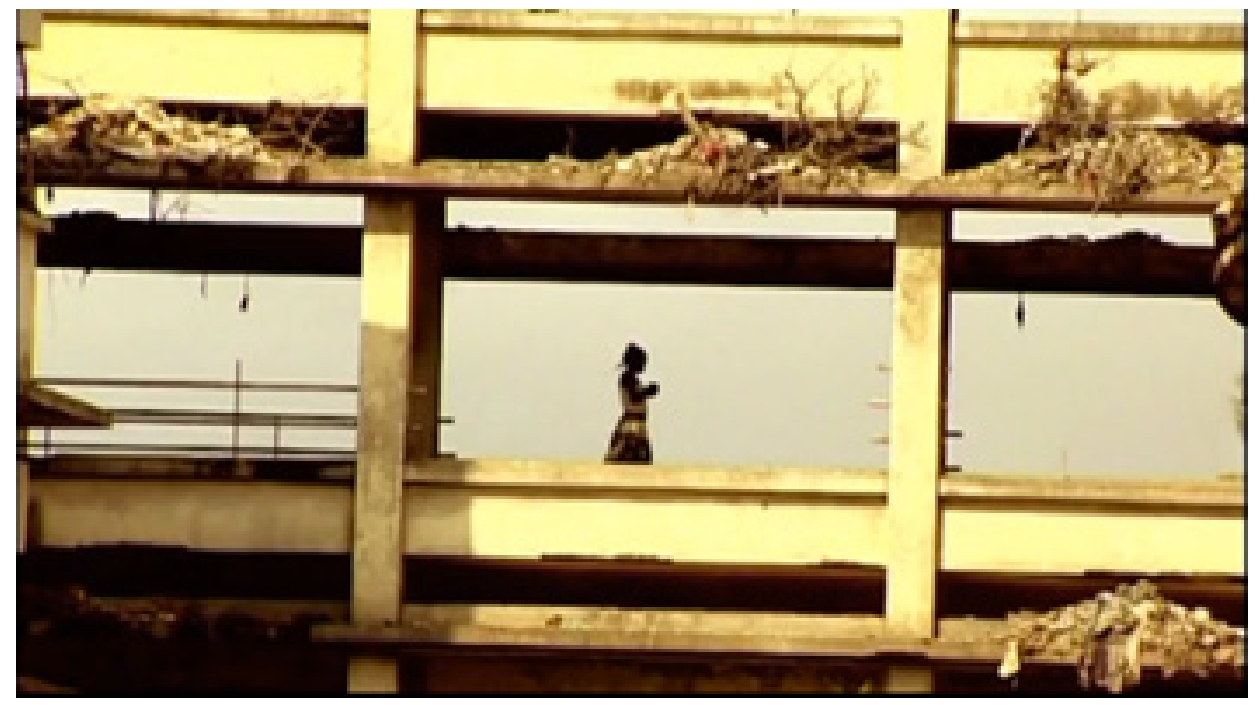

Abb. 2: Fehlende Fenster II.

Quelle: HÓSPEDES, 2007, 00:04:04. 
Im Darbieten der Leerstellen wird schon symbolhaft die Verwundbarkeit der Gesellschaft ausgestellt, die in diesem Betonskelett den Gezeiten ausgesetzt ist. Man kann sich hier „drinnen“ weder vor Wetter noch vor Eindringlingen schützen. Das Fenster, das normalerweise durch seine Lichtdurchlässigkeit Räume wohnlicher macht, trägt hier zu dem genauen Gegenteil bei; das Fehlen von Fenstern kann verheerende Folgen haben, wie Übergriffe, Unfälle von Betrunkenen, Stürze von Kindern. Dadurch, dass anstatt der Fenster nun Leerstellen stehen, wirkt das gesamte Gebäude wie ein einziger Durchgang. Es wird zwar durch Türen versucht Privatheit herzustellen, insgesamt ist das Hotel jedoch eine einzige große Passage, ein Wohnort im ewigen Transit.

Ein weiteres bildhaftes Beispiel des Übergangs ist die Treppe: In Grande Hotel (2010) wird Moises interviewt, der sich und seiner Familie im Grande Hotel ein Heim geschaffen hat. Dabei erklärt er der Kamera, wie er den Treppenabschnitt in eine Wohnung umfunktioniert hat. Aus der Not heraus erfand er diesen Wohnraum, verwandelte die Begegnungszone „Treppe“ in einen privaten Innenraum, um seiner Familie ein Heim zu bieten (GRANDE, 2010, 00:07:28 - 00:08:38). Doch obwohl sich seine Familie hier beheimatet hat, ist sich Moises der Instabilität seiner Wohnsituation bewusst. Denn auch wenn er Interesse daran hat, die Lebensqualität von sich, seiner Familie und der gesamten Gemeinschaft des Grande Hotel zu verbessern, weiß er, dass sie vom Rest der Gesellschaft als ein Kollektiv von Kriminellen gesehen werden, deswegen exkludiert sind und niemals auf staatliche Unterstützung hoffen können, um das baufällige Gebäude vor dem unvermeidlichen Einsturz zu retten.

Das wohl häufigste und aussagekräftigste filmische Beispiel des Liminoiden ist in beiden Filmtexten die Tür. Insbesondere Azevedo spielt in Hóspedes da Noite (2007) mit den Möglichkeiten, die dieser Übergangsbereich als filmisches Mittel, als Metapher bietet. Türen - genauso natürlich wie Fenster - sind Verbindungsglieder zwischen der äußeren Welt und dem Inneren des Zuhauses, zwischen Öffentlichkeit und Privatsphäre. Als Übergänge stellen sie eine Beziehung zwischen den beiden Welten her. Während das Fenster sowohl den Blick hinaus als auch den Blick hinein bietet, hat die Tür einen semipermeablen Charakter (BOLLNOW 1959, p.113). Sie kann als Instrument der Kontrolle dienen, da man durch sie die Macht erlangt, andere aus seiner Privatheit auszuschließen; umgekehrt bietet sie auch die Möglichkeit, Gastfreundschaft zu demonstrieren, sowie natürlich die Freiheit, in die Welt zu schreiten.Denn mit dem Durschreiten der Tür hängt auch immer das Überschreiten der Schwelle zwischen Drinnen und Draußen zusammen (BOLLNOW 1959, p. 115). In Hóspedes da Noite (2007) wird dieses Hinaustreten in die Welt inszeniert, indem die Kamera ihren Blick auf die Personen richtet, die das Gebäude verlassen, selbst aber im Hotel zurückbleibt (00:27:46; 00:27:59). 
Durch die Tür tritt man ebenso in den Lebensbereich eines anderen Menschen. Insbesondere die dokumentarische Kamera eignet sich für dieses Hineinblicken in das Leben der Personen. Sie kann von den Bewohner:innen hineingebeten werden, aber auch - durch den Einsatz filmischer Mittel - in fremde Lebenswelten eindringen. Hier zeigt sich der eigentliche narrative Gehalt des Liminoiden: Beide Filme spielen mit diesen Schnittstellen, um die Einzelschicksale hinter den vielen Lebenswelten aufzudecken und sie zu einer vielschichtigen Erzählung der nationalen Geschichte zu verknüpfen. Das Mosaik an Türen, das sich insbesondere in Hóspedes da Noite (2007), aber auch in Grande Hotel (2010) finden lässt, kann als Metapher für das Mosaik an (potenziellen) Geschichten gelesen werden, die sich nicht nur in diesem Moment, sondern zu allen Zeiten dahinter abgespielt haben. Hinter ihnen überlagern sich Räume und Erinnerungen, die sich am Ort des Hotels und seiner Räume eingeschrieben haben. Das Mosaik der Erzählungen der Bewohner:innen, der ehemaligen Gäste sowie der Angestellten erweitert sich auf ein Mosaik der Bilder. Es wird oftmals von der Tür auf den Raum dahinter geblendet oder geschnitten, aber nicht immer; Türen und Lebenswelten öffnen sich (Abb. 3), bleiben aber auch verschlossen (Abb. 4). Aus diesem Grund ist die Rede von vielen kleinen, potenziellen Geschichten: Hier wird zusätzlich das Spiel zwischen Einblick und Ausschluss, Privatheit und Öffentlichkeit visuell repräsentiert.

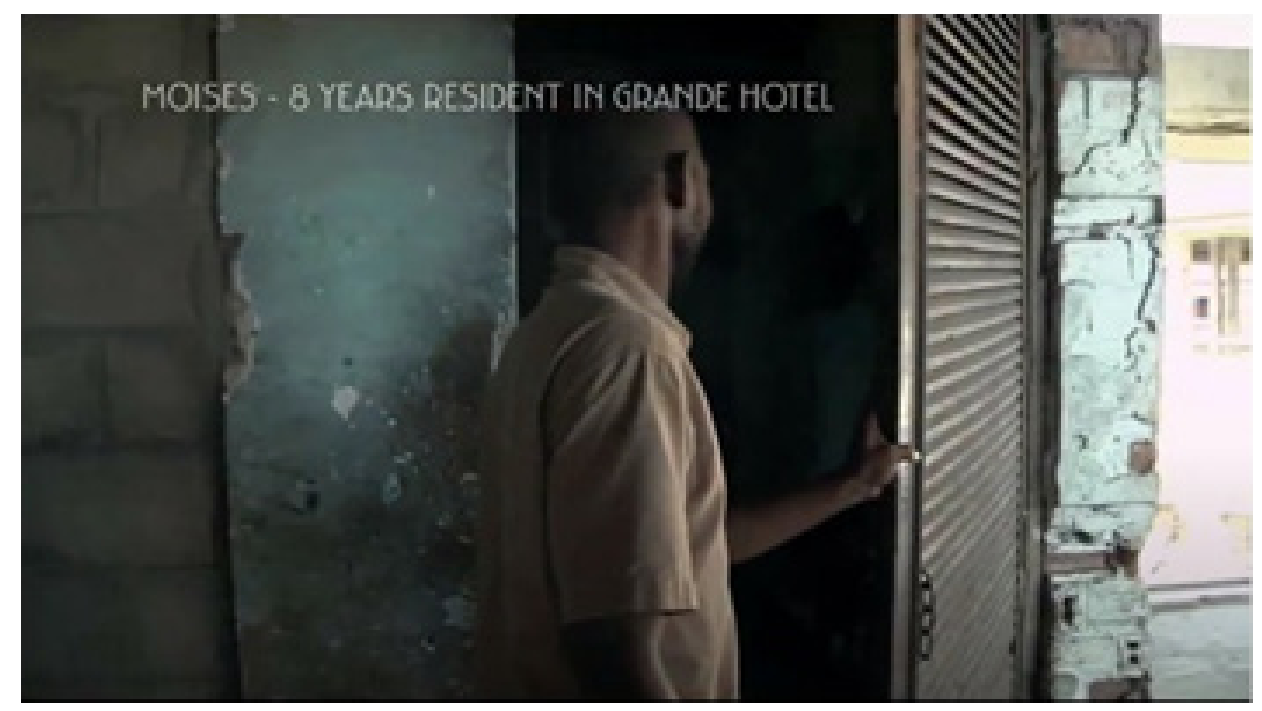

Abb. 3: Moises lädt die Kamera ein.

Quelle: GRANDE, 2010, 00:07:34. 


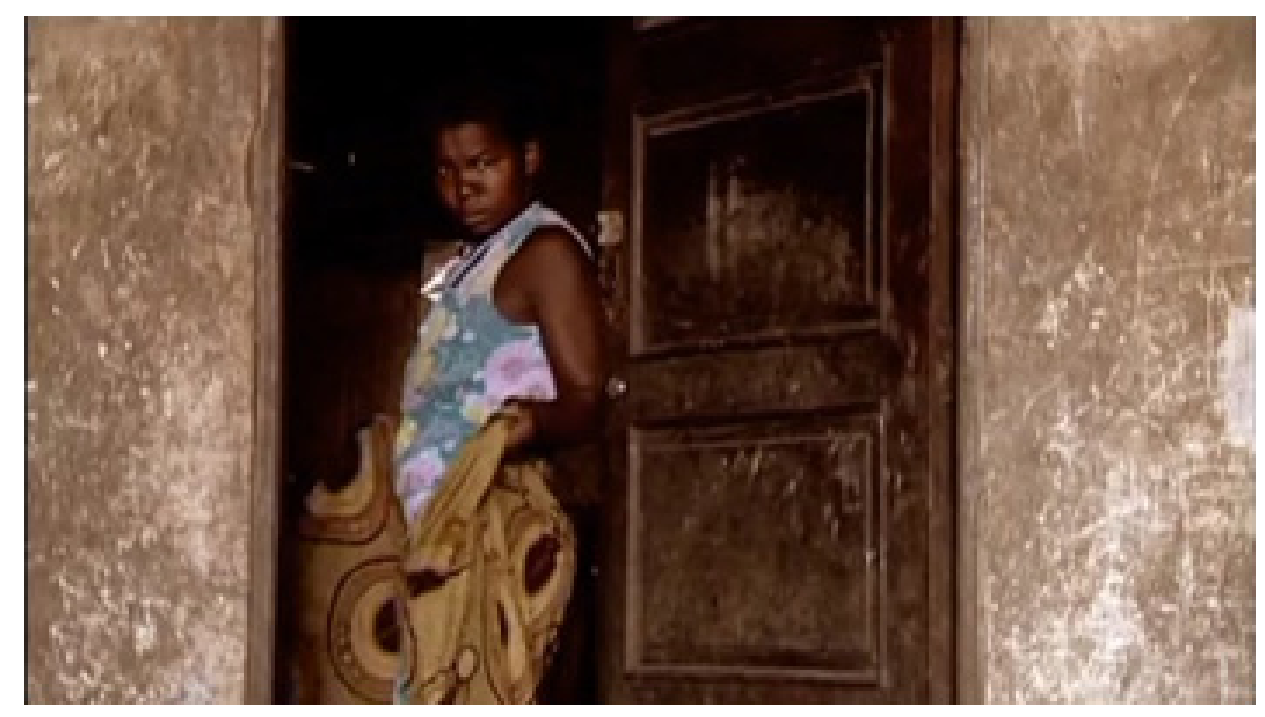

Abb. 4: Tür wird geschlossen.

HÓSPEDES, 2007, 00:10:0

\section{VIELSTIMMIGE „ERZÄHLUNG“ VON ERINNERUNG}

Die Vielstimmigkeit der Erzählungen und Erinnerungen wird durch den Einsatz unterschiedlichster filmischer Strategien der Bild- und Tonmontage ermöglicht, die das Dokumentarische mit dem Fiktionalen vermengen ${ }^{5}$. Die beiden Filmemacher bedienen sich hierzu einer Vielzahl an Mitteln audiovisueller Sinnstiftung (z.B. Überblendung (Abb. 5-7), scharfe Schnitte (Abb. 8-9), Stimmen und Musik aus dem Off etc.), die einzelnen Fragmente von Gegenwart und Vergangenheit innerhalb ihres Erzählkontinuums hervorholen und in einen gänzlich neuen Gesamtzusammenhang bringen. Visuelle Kontrastierungen verschiedener Zeitschichten überhöhen ihrerseits noch die Vielstimmigkeit der erzählten und erinnerten Fragmente.

Diese Art der dokumentarfilmischen Montage bedeutet keine Abweichung, sondern vielmehr eine sinngebende Ergänzung der Realität (HICKETHIER, 2001, p. 145): Durch die Summe der Bilder in ihrer filmischen Form entsteht eine Bedeutung, die letztlich von der Rezeption und Deutung der Zusehenden abhängt. Diese Bedeutung speist sich aus den Erinnerungen sowohl der Bewohner:innen des Grande Hotel, als auch aus denjenigen der Rezipient:innen der Filme - angereichert mit ihren jeweiligen kolonialen und (post-)kolonialen Perspektiven, Beziehungen, Geschichten. Diese werden immer wieder gebrochen, durch die ",dokumentarischen“ Schilderungen der beiden alten Führer (HÓSPEDES, 2007) sowie durch die „live“ erzählten Lebens-Geschichten der aktuellen Bewohner:innen, und bilden damit ein Mosaik aus sich überlagernden, sich gegenstrebig widersprechenden und gleichzeitig bereichernden Mosaiksteinen der Memoria.

Der soziohistorische Hintergrund des Grande Hotel und einhergehend damit die Kontrastierung von Vergangenheit und Gegenwart wird u.a. durch den Einsatz von originalem Bildmaterial hervorgehoben. Während 
Lotte Stoop durchgängig davon Gebrauch macht, wendet Licínio Azevedo diese Strategie nur zu Beginn an, indem er Bilder inszeniert, die das Hotel in ihrem früheren Zustand zeigen, um sie durch Überblenden mit den jetzigen Bildern zu kontrastieren:

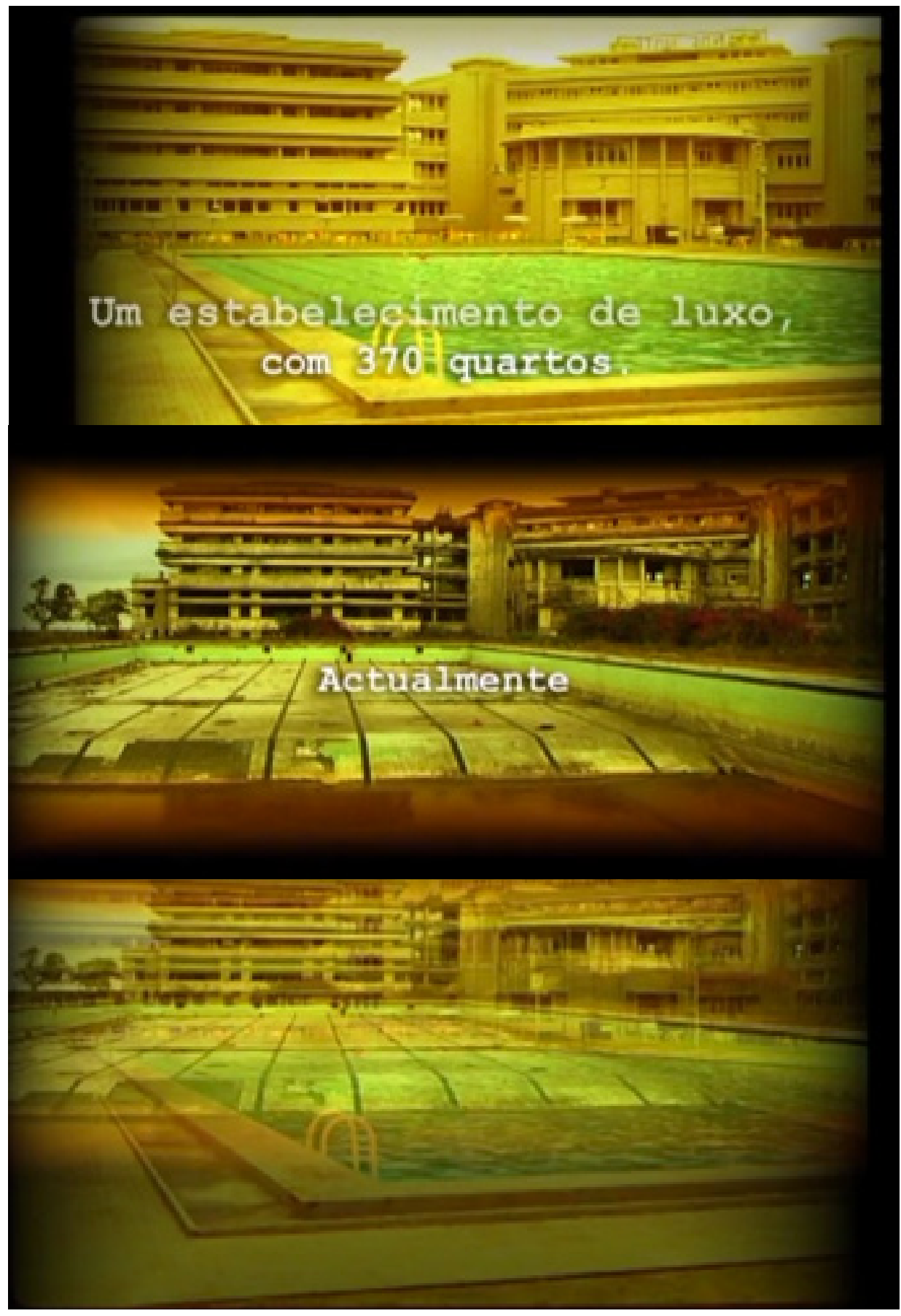

Abb. 5: Das Hotel im Jahr 1953.

Quelle: HÓSPEDES, 2007, 00:00:48.

Abb. 6: Blende von damals zu heute.

Quelle: HÓSPEDES, 2007, 00:00:53.

Abb. 7: Das Hotel im Jahr 2007.

Quelle: HÓSPEDES, 2007, 00:00:57. 
Azevedo stellt mit dieser Bildmontage von Beginn an den historischen Kontext her, der sich durch den ganzen Film zieht. Dabei wird zusätzlich zur historischen Vielschichtigkeit dieses Ortes die räumliche Geschichtlichkeit hervorgehoben. Durch schnelle Schnitte und Fragmentierung der Erzählungen erwirkt der Film ein gesamtes Soziogram der mosambikanischen Bevölkerung in ihrer (postkolonialen) Polyphonie.

Stoops setzt vor allem auf die Hervorhebung von Gegensätzen durch den prominenteren Einsatz von faktualen Bildern und Videos der damaligen Dekadenz. Sie verwendet dabei keine Blenden, sondern harte Schnitte, gelangt aber zu einem ähnlichen Ergebnis:

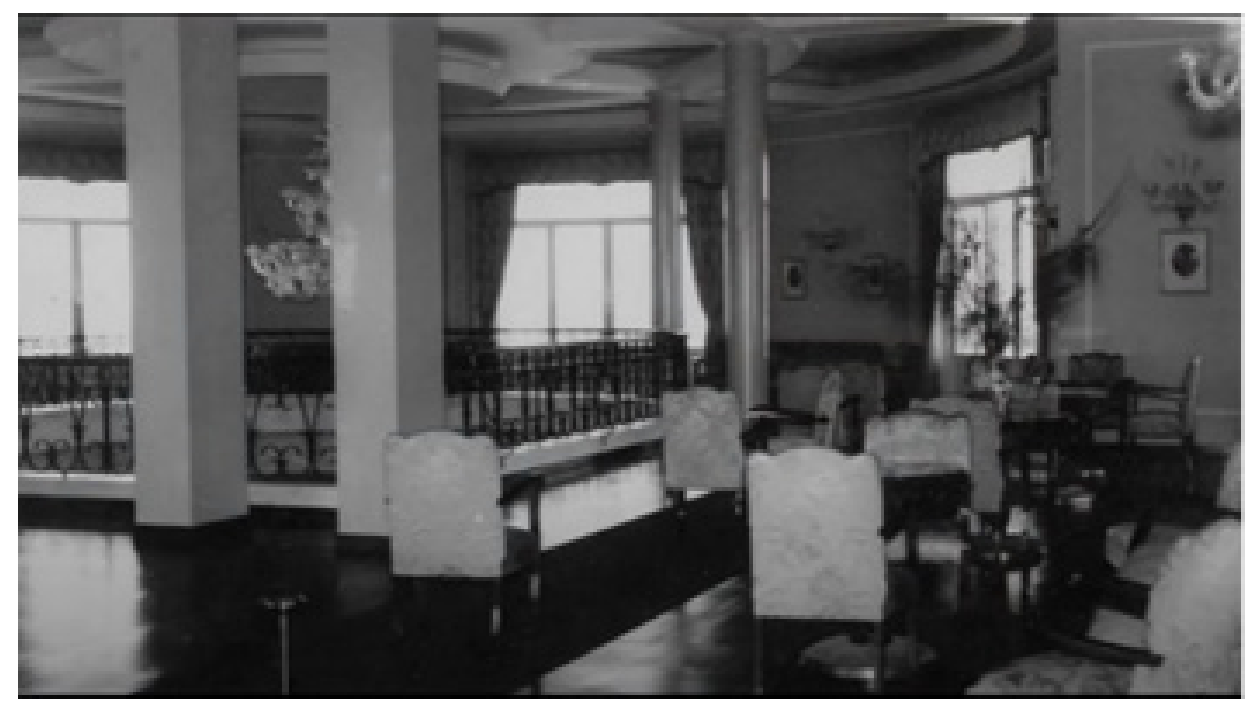

Abb. 8: Grande Hotel früher.

Quelle: GRANDE, 2010, 00:47:04.

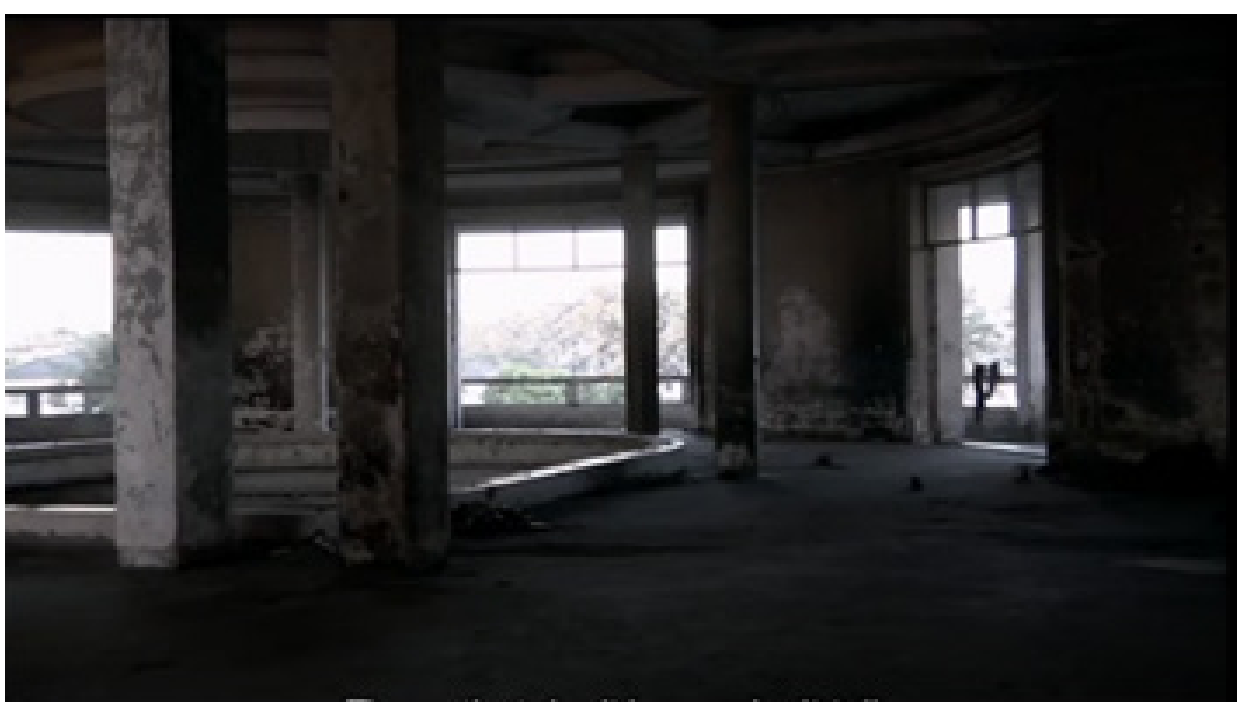

Abb. 9: Grande Hotel heute.

Quelle: GRANDE, 2010, 00:47:11. 
Auch in Grande Hotel (2010) wird als ein Mosaik an Geschichten, Bildern und Stimmen inszeniert. Diese wirken jedoch stärker emotionalisiert, was besonders durch die Bild-Ton-Konstellation und den Einsatz von Filmmusik erreicht wird.

Die in beiden Filmen gezeigten Interviews tragen nicht nur zum „authentischen" Charakter bei, indem sie den „realistischen“ Eindruck unterstreichen sollen, sondern heben auch die Bedeutung der Pluralität und Vielstimmigkeit hervor.Wie bereits erwähnt, bedienen sich auch dokumentarische Filme dramaturgischer Eingriffe, um bestimmte Zusammenhänge sichtbar zu machen, die ansonsten nicht wahrnehmbar wären. Kurze Sequenzen und ein mosaikartiger Schnitt verweben in beiden Filmen die individuellen Erinnerungen zu einer kollektiven Geschichte. Indem die individuellen Schicksale als Fragmente erzählt werden, die sich in der Geschichte eines von Kolonialismus und Krieg zerrütteten Landes verlieren, bekommen sie durch den dokumentarischen Film wieder Bedeutung zugeschrieben.

Eine Brücke in die Vergangenheit schlägt Hóspedes da Noite (2007) immer wieder mithilfe der ehemaligen Hotelangestellten, die in gewisser Weise die Rolle der Erzähler übernehmen. Die Aufmerksamkeit des Publikums wird dadurch scheinbar mit einem Minimum an filmischer Intervention gelenkt. Dabei hilft der Zeigegestus, mit dem die beiden Männer in nostalgischer Retrospektive auf die Vergangenheit der einzelnen Räume verweisen, den Zuschauer:innen, der Logik der Erzählung - also der intendierten Kontrastierung von Vergangenheit und Gegenwart - zu folgen. Zusätzlich betont die Kamera ihren Status als Zeitzeugen, sowie die bezeugende Funktion und die Authentizität des Dokumentarischen.

Der Film Grande Hotel (2010) bindet die Stimmen der Vergangenheit fast ausschließlich in Form von Voice-Overs aus dem Off ein. So werden die visuellen Eindrücke der Gegenwart mit den Stimmen der Vergangenheit überlagert, um die historische Vielschichtigkeit, das heißt die Geschichte, die sich in der Gegenwart ausdrückt, zu veranschaulichen. Die gesichtslosen Stimmen schaffen die Ästhetik träumerischer Erinnerung, wodurch die nostalgische Romantisierung aufgedeckt wird: durch die kontrastierende Gegenüberstellung von heutigen Wohnräumen und damaligen Hotelgeschichten wird das Paradox dieser Zeitschichten filmisch ausgestellt. Das narrative Umherschweifen zwischen diesen Zeitebenen bringt koloniale Dekadenz und gegenwärtigen Zerfall in eine Juxtaposition, indem sich unterschiedlichstes Bildmaterial, extradiegetische Musik und Erzählungen aus dem Off asynchron überschneiden und in einen neuen Sinnzusammenhang bringen (z.B. GRANDE, 2010, 00:21:33-00:22:00).

Der melancholische Duktus des Rückblicks wird von Grande Hotel (2010) auch mithilfe von Geräuschen inszeniert. So wird das Gefühl von Sehnsucht und Heimweh, die saudade, die Berta, eine retornada, also eine „Zurückgekehrte“, verspürt, zu Beginn des Films bereits durch das Rauschen des Meeres angekündigt. Der Film arbeitet hier mit zwei eng verbundenen, semantisch geladenen Elementen der portugiesischen Kunst und Kultur - dem Meer, das im Laufe des Films immer wieder gezeigt wird, 
und der saudade -, um auf das Thema der Heimat bzw. der Heimatlosigkeit zu verweisen.Dies wird gegen Ende des Films durch eine Aussage Bertas unterstrichen: „The Portuguese people have their own sea. It's not bad, but it's not mine." (GRANDE, 2010, 01:06:27-01:06:32). Durch die Inszenierung des nostalgischen Rückblicks wird der enorme Einflusshervorgehoben, den die Kolonial-Politik im persönlichen Leben haben kann, vor allem in Bezug auf das grundlegendste menschliche Bedürfnis: das Gefühl, Zuhause zu sein.

\section{KONKLUSION}

Das ästhetische Wechselspiel nimmt - bei all seiner dokumentarischen Aufbereitung - dann den Filmen auch wieder ihren dokumentarischen Charakter. Denn es sind gerade diese Mini-Geschichten, von denen die Filme leben, die sie inszenieren, die sie visualisieren, die sie speichern und damit der Gesellschaft quasi als Archive der kollektiven Memoria nachhaltig zur Verfügung stellen. Die Vielfalt der Narrationen, die sich in den Fluren, Gängen, hinter den Türen und Fenstern verbergen, machen die Filme zu polyphonen, nachgerade postmodernen Erzähltexten, die eben nicht die eine Version der Geschichte anvisieren, vielmehr eine Re-Evaluierung der Geschichte durch die kleinen Ich-Geschichten (WELSCH, 2002), eine Art Revision der Geschichte anregen.

Über die filmische Inszenierung des ehemaligen Kolonial-Hotels wird nicht nur ein Geschichts- und Wohnraum abgebildet, dieser wird darüber hinaus zu einer Projektionsfläche gesellschaftlicher Diskurse umfunktioniert. Durch den soziokulturellen Prozess des doku-filmischen Erzählens der Bewohner:innen wird dem Nicht-Ort Hotel - beziehungsweise Flüchtlingslager - Identität und Geschichte eingeschrieben. Anstatt sie als ein stereotypisiertes Kollektiv von Kriminellen und Flüchtlingen abzubilden, gibt der Film ihnen die Möglichkeit zur Individualisierung und Menschlichkeit zurück, indem er die Vielstimmigkeit dieses Ortes inszeniert. Während die Authentizitätsmerkmale den Realitäts- und damit Identifikationseffekt verstärken, ermöglicht die konstruktive Bild- und Tonmontage die Verknüpfung von Vergangenheit und Gegenwart und damit die Auseinandersetzung mit individueller ebenso wie kollektiver (post)kolonialer Memoria, und zwar über eines der zentralsten menschlichen Bedürfnisse: der Beheimatung.

\section{BIBLIOGRAFIE}

\section{PRIMÄRTEXTE}

GRANDE Hotel. Regie: Lotte Stoops. Produktion: Serendipity Films / Volya Films. Belgien: Serendipity Films, 2010. (70 min.). URL: https://www. serendipityfilms.be/grande-hotel. Letzter Zugriff: 24.05.2021.

HÓSPEDES da Noite. Regie: Licínio Azevedo. Produktion: Ébano Multimedia. Moçambique : Marfilmes, 2007. 1 DVD. (53 min.). 


\section{SEKUNDÄRTEXTE}

AUGÉ, Marc. Orte und Nicht-Orte. Vorüberlegungen zu einer Ethnologie der Einsamkeit. 2. Auflage. Übersetzung.: Michael Bischoff. Frankfurt a. M.: Fischer Verlag, 1994. Original: Non-Lieux. Introduction à une anthropologie de la surmodernité.

AUMONT, Jacques. L'image. Paris: Nathan, 1990.

BARTHES, Roland. Le bruissement de la langue. Essais critique IV. Paris : Éditions du Seuil, 1984.

BLUM, Philipp. Doku-Fiktionen: Filme auf der Grenze zwischen Fiktion und Non-Fiktion als ästhetische Interventionen der Gattungslogik. MEDIENwissenschaft, H. 2, p. 130-144, 2013.

BOLLNOW, Otto Friedrich. Der Mensch in der Spannung zwischen öffentlicher und privater Sphäre. In: PROHASKA, Leopold; HAIDER, Franz (org.). Jugendgemäße Lebenskunde in der Entscheidung. Wien: Österr. Bundesverl. für Unterricht, Wissenschaft und Kunst, 1970. p. 17-27.

. Der Mensch und der Raum. Universitas, 18. Jg., p. 499-514, 1963.

Tür und Fenster. Die Sammlung, 14. Jg., p. 113-120, 1959.

DÜLLO, Thomas. Wohnen im Film. In: SCHROER, Markus (org.), Gesellschaft im Film. Konstanz: UVK Verl.-Ges., 2008. p. 357-393.

GESCHKE, Sandra Maria. Doing Urban Space: Ganzheitliches Wohnen zwischen Raumbildung und Menschwerdung. Bielefeld: transcript, 2013.

GRANDE HOTEL. Regie: Anabela de Saint-Maurice. Produktion: Ana Lucas. Portugal: RTP, 2007 (52 min.). URL: https://www.cultureunplugged. com/documentary/watch-online/play/7430/Grande-Hotel. Letzter Zugriff: 24.05.2021.

HATTENDORF, Manfred. Dokumentarfilm und Authentizität: Ästhetik und Pragmatik einer Gattung. 1. Auflage. Konstanz: Ölschläger, 1994.

HIßNAUER, Christian. MöglichkeitsSPIELräume: Fiktion als dokumentarische Methode. Anmerkungen zur Semio-Pragmatik fiktiver Dokumentationen. MEDIENwissenschaft, H. 1, p. 17-28, 2010.

HICKETHIER, Knut. Film- und Fernsehanalyse. 3. überarb. Auflage. Stuttgart: J.B. Metzler, 2001.

KIENER, Wilma. Die Kunst des Erzählens: Narrativität in dokumentarischen und ethnographischen Filmen. Konstanz: UVK-Medien, 1999.

MUNDHENKE, Florian. Zwischen Dokumentar- und Spielfilm: Zur Repräsentation und Rezeption von Hybrid-Formen. Wiesbaden: Springer, 2017.

PUTSCH, Christian. Wenn aus Luxus-Hotels Geisterhäuser werden. Welt, 2010. URL: https://www.welt.de/reise/Fern/article11526639/Wenn-ausLuxus-Hotels-Geisterhaeuser-werden.html. Letzter Zugriff: 24.05.2021. 
SPINUZZA, Giulia. O ciclo do Grande Hotel da Beira: os documentários Grande Hotel, de Lotte Stoops; Grande Hotel, de Anabela Saint-Maurice; Hóspedes da Noite, de Licínio Azevedo e Amanhecer a Andar, de Sílvia Firmino. Remate de males, Campinas-SP, v. 38, n. 1, p. 161-185, 2018.

Olhares cruzados sobre o Grande Hotel da Beira: Hóspedes da Noite, de Licínio Azevedo, e ,Casa de Ferro', de João Paulo Borges Coelho. Cerrados, v. 41, p. 276-287, 2016.

WELSCH, Wolfgang. Unsere postmoderne Moderne. 7. Auflage. Berlin: Akademie Verlag, 2002.

Recebido para avaliação em 31/05/2021

Aprovado para publicação em 26/07/2021

\section{NOTAS}

1 Kathrin Sartingen é Professora Titular de Literaturas, Culturas e Mídias em língua portuguesa e espanhola (com enfoque especial em América Latina e África) no Instituto de Línguas e Literaturas Românicas da Universidade de Viena, Áustria. Até 2019 ela foi Presidente da Associação Alemã de Lusitanistas (Deutscher Lusitanistenverband - DLV). Suas áreas de pesquisa são: Relações literárias, fílmicas e culturais transatlânticas (Iberia - África - América Latina); Literaturas e filmes africanos e latino-americanos; Intertextualidades, Intermidialidades e interculturalidades (cine, teatro, literatura); Estudos culturais e Teorias pós-coloniais.

2 Sophie Baltas é estudante de mestrado de Letras Românicas com enfoque em literaturas, culturas e filmes dos países da língua portuguesa na Universidade de Viena, Áustria. Licenciatura em Estudos de Teatro, Filmes e Mídias. Suas áreas de pesquisa são: literaturas e filmes africanos, brasileiros e portuguêses; estudos culturais e sociais (urbanização, espaços habitacionais, memória, pós-colonialismo).

3 Die genaue Zahl der Bewohner ist verständlicherweise schwer zu bestimmen, da diese nicht offiziell im ehemaligen Hotel wohnen. Die Zahlen in der angeführten Literatur und den beiden Filmen bewegen sich zwischen 2.000 und 3.000 Personen (GRANDE HOTEL, 2007; GRANDE HOTEL, 2010; HÓSPEDES DA NOITE, 2007; PUTSCH, 2010).

4 Dieser Realitätseffekt geht auf den effet du réel von Roland Barthes (1984) zurück.

5 Durch die Bearbeitung des filmischen Materials und damit der Verfolgung einer bestimmten Erzählstrategie gelangt der ,fiktionale“ Dokumentarist „(...) zu den tiefer liegenden, verborgenen, vermittelten, unmittelbar nicht wahrnehmbaren Zusammenhängen der gesellschaftlichen Wirklichkeit“ (KIENER, 1999, p. 169). Azevedos Filme bewegen sich stets in eben dieser Sphäre zwischen fiktional und dokumentarisch (SPINUZZA, 2016, p. 277); diese Tendenz findet sich ebenso in Stoops' Film. 